\title{
A study on correlation between strain effect and conduction mechanism of $\mathrm{La}_{0.9} \mathrm{Ca}_{0.1} \mathrm{MnO}_{3}$ films
}

\author{
S. Uthayakumar ${ }^{1 *}$, P. Santhosh ${ }^{2}$, G. H. Aydogdu, H. -U. Habermeier \\ Max Planck Institute for Solid State Research, Heisenbergstrasse 1, D-70569 Stuttgart, Germany \\ *Corresponding author. Tel.: (+44) 178441 4053; Fax: (+44) 178447 2794; E-mail: s.uthayakumar@rhul.ac.uk \\ ${ }^{1}$ Presently at Department of Physics, Royal Holloway University of London, Egham, Surrey TW20 OEX, UK \\ ${ }^{2}$ Presently at Microsensors for Clinical Research and Analysis, Institute of Technology Tallaght, Dublin 24, Ireland
}

Received: 11 March 2013, Revised: 10 May 2013 and Accepted: 12 May 2013

\section{ABSTRACT}

Thin films of $\mathrm{La}_{0 .} \mathrm{Ca}_{0.1} \mathrm{MnO}_{3}$ with thicknesses in the range of 40-200 nm grown on (100) SrTiO employing pulsed laser deposition (PLD) technique have been investigated by measuring structure and transport properties. The structural properties of the films were studied by X-ray diffraction (XRD) technique employing $2 \theta-\omega$ scan and the results show a high quality crystalline nature with ' $b$ ' axis orientation. The Jahn-Teller strain $\left(\varepsilon_{\mathrm{J}-\mathrm{T}}\right)$ was found to decrease with thickness. Transport measurements were performed using standard four probe technique, which shows an insulating behavior for all the thickness at low temperatures. The high temperature resistivity obeys the small polaron hopping. Further more, the obtained results from the transport measurement shows lowering of polaron binding energy for the films grown under tensile strain as compared to our previous work on $\mathrm{La}_{0.9} \mathrm{Sr}_{0.1} \mathrm{MnO}_{3}$ films.

Keywords: Perovskite manganites; polycrystalline ceramics; magnetoresistance effect; transport mechanism.

\section{Introduction}

Since the discovery of colossal magnetoresistance in hole doped perovskite type $\mathrm{A}_{1-\mathrm{x}} \mathrm{B}_{\mathrm{x}} \mathrm{MnO}_{3}$ (where $\mathrm{A}=\mathrm{La}^{3+}, \mathrm{Pr}^{3+}$, $\left.\mathrm{Nd}^{3+} \& \mathrm{~B}=\mathrm{Sr}^{2+}, \mathrm{Ca}^{2+}, \mathrm{Ba}^{2+}\right)$, substantial progress in understanding the electronic and magnetic properties has been made [1]. It has been demonstrated that the oxides of perovskite manganites show a significant difference in the transport properties based on the type of the samples such as thin films, single crystals and polycrystalline ceramics. In the case of polycrystalline material, the presence of grain boundaries leads to a large magnetoresistance effect over a wide range of temperature below the curie temperature $\left(\mathrm{T}_{\mathrm{c}}\right)$. However, in the case of single crystalline or thin films, the large magnetoresistance effect is found to exist in a relatively narrow temperature around $\mathrm{T}_{\mathrm{c}}$. Since commercial applications require these materials as thin films rather than bulk or single crystals, a detailed knowledge of electronic and magnetic properties is mandatory to exploit the potential use of the manganites.

The substitution of trivalent rare earth ions by divalent rare earth alkaline ions creates mixed $\mathrm{Mn}(\mathrm{III}) / \mathrm{Mn}(\mathrm{IV})$ state resulting in mobile charge carriers and this in turn influences the physical properties. Double exchange mechanism and Jahn-Teller effect successfully explains the physical properties of these manganese oxides [2]. The basic feature of double exchange mechanism entangles with the transfer of ' $\mathrm{e}_{\mathrm{g}}$ ' electrons between $\mathrm{Mn}^{3+}$ and $\mathrm{Mn}^{4+}$ ions. During the transfer, the carrier avoids strong on site Hund exchange energy and facilitates hopping process. Along with the transfer of ' $\mathrm{e}_{\mathrm{g}}$ ' electrons it accompanies lattice distortions which have been explained well by JahnTeller effect. Such Jahn-Teller distortion around $\mathrm{Mn}^{3+}$ localizes the charge carriers and gives rise to polaron formation [3]. The existence of polarons has been confirmed from many experimental results and reported elsewhere [4-6].

In general, the polaronic state emerges due to the consequence of strong electron-phonon coupling of the Jahn-Teller active $\mathrm{Mn}^{3+}$ ions. To get deep insight into the polaronic behavior and to exploit the mechanism causing the semiconductor like transport properties at higher temperature, a large number of models have been proposed. The models include thermal activation, variable-range hopping and adiabatic small polaron conduction [7]. In most cases for the observed transport properties, the hopping conduction of small polarons in the adiabatic approximation is accounted [8]. Hence, using this adiabatic approximation, investigation was made on the conduction mechanism of $\mathrm{La}_{0.9} \mathrm{Ca}_{0.1} \mathrm{MnO}_{3}$ thin films in the light of our previous study on $\mathrm{La}_{0.9} \mathrm{Sr}_{0.1} \mathrm{MnO}_{3}$ films, where the strain dependence of the electronic transport properties and the thickness dependence of the polaron formation energy was 
studied [9]. The present study emphasis how the transport mechanism behaves in a different strain states as a consequence of strain effect. At lower substitution level, the competition between electronic and magnetic properties drives the phase separated state to be a stable ground state. In this study, we used low level doped high quality epitaxial $\mathrm{La}_{0.9} \mathrm{Ca}_{0.1} \mathrm{MnO}_{3}$ films grown by Pulsed laser deposition technique deposited on (100)-oriented $\mathrm{SrTiO}_{3}$ substrate.

\section{Experimental}

Thin films of $\mathrm{La}_{0.9} \mathrm{Ca}_{0.1} \mathrm{MnO}_{3}$ with different thicknesses were deposited on (100) oriented $(\mathrm{a}=3.905 \AA) \mathrm{SrTiO}_{3}$ substrate using pulsed laser deposition technique. The deposition process was performed in a pure oxygen gas of pressure 0.405 mbar. The substrate temperature was kept at $800^{\circ} \mathrm{C}$ during the growth. An excimer laser (Lambda Physik) with the laser fluency of $1.6 \mathrm{~J} \mathrm{~cm}^{-2}$, wavelength of $248 \mathrm{~nm}$ with pulse frequency of $5 \mathrm{~Hz}$ was employed. To maintain the oxygen stoichiometry after deposition, the as grown films were subjected to annealing in air at $900^{\circ} \mathrm{C}$ for $30 \mathrm{~min}$. The thickness of the films was controlled by calculating the ablation period during the growth process.

The as grown thin films were subjected to various characterization techniques. The structural properties of the as grown films were studied by Bruker D8 X-ray diffractometer in the mode of $2 \theta-\omega$ scan with $\mathrm{Cu} K \alpha$ radiation $(\lambda=1.54056 \AA)$. Transport properties were measured using standard four-probe method. To establish electrical contacts, evaporated chromium-gold pads and silver epoxy were used to attach the gold wires to the film and DC current was kept at $10^{-7} \mathrm{~A}$ during the measurement. The obtained results are compared for the various thickness to validate the adiabatic small polaron hopping model.

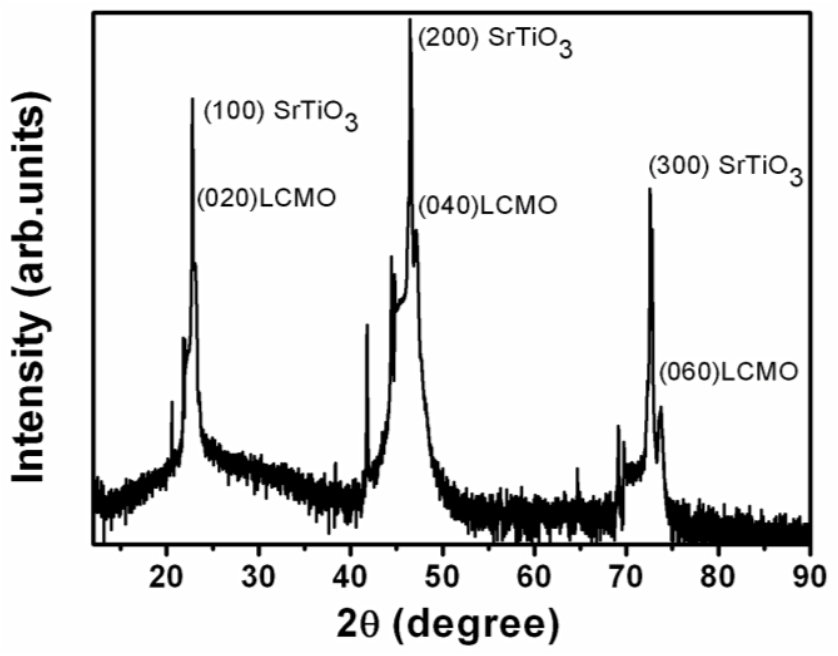

Fig. 1. 2 $\theta-\omega$ scan of $40 \mathrm{~nm}$ thick $\mathrm{La}_{0.9} \mathrm{Ca}_{0.1} \mathrm{MnO}_{3}$ films.

\section{Results and discussion}

The XRD diffractogram $(2 \theta-\omega$ scans $)$ for the $\mathrm{La}_{0.9} \mathrm{Ca}_{0.1} \mathrm{MnO}_{3}$ film $(40 \mathrm{~nm})$ is shown in Fig. 1. No other parasitic peaks are observed besides the reflections from the substrate and (010) reflections of the film. The evaluated cell parameters demonstrate that $\mathrm{La}_{0 .} \mathrm{Ca}_{0.1} \mathrm{MnO}_{3}$ possess orthorhombic crystal structure with $\mathrm{P}_{\mathrm{nma}}$ space group with a tensile in-plane epitaxial strain and a detailed lattice parameter values for series of $\mathrm{La}_{0.9} \mathrm{Ca}_{0.1} \mathrm{MnO}_{3}$ evaluated by Nelson-Riley function [10]. For the $40 \mathrm{~nm}$ film, the cell volume is calculated to be $234.41 \AA$, which is smaller by $0.83 \%$ compared to the bulk cell volume $(236.37 \AA)$. However, in the case of $200 \mathrm{~nm}$, an increase in cell volume of about $0.07 \%$ is observed. It is presumed that the variation of cell volume might be due to the differences in the oxygen content for various thicknesses. The mixed valence state of $\mathrm{Mn}^{3+} / \mathrm{Mn}^{4+}$ caused by the substitution of $\mathrm{Ca}^{2+}$ by $\mathrm{La}^{3+}$ develops orthorhombic distortion which is measured by the asymmetry parameter $\delta$ $=\left(\sqrt{ } 2 c /\left(a^{2}+b^{2}\right)^{1 / 2}\right)_{-1}$, Fig. 2 .

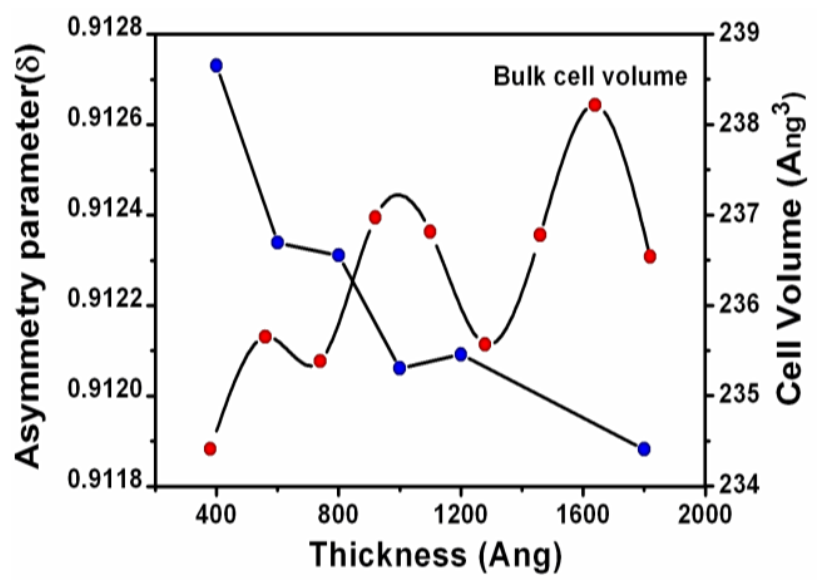

Fig. 2.Variation of the asymmetry parameter and cell volume of $\mathrm{La}_{0.9} \mathrm{Ca}_{0.1} \mathrm{MnO}_{3}$ films with thickness.

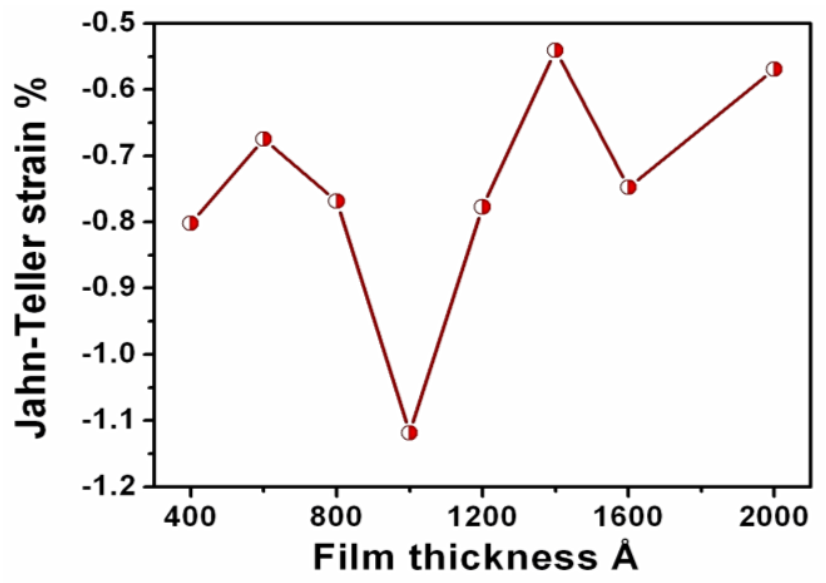

Fig. 3. Jahn-Teller strain versus thicknesses of the $\mathrm{La}_{0.9} \mathrm{Ca}_{0.1} \mathrm{MnO}_{3}$ films.

It is evident that a reduction of ' $\delta$ ' is observed for orthorhombic distortion, which in turn facilitates the enhancement of Mn-O-Mn bond angle close to the ideal value $180^{\circ}$. The cell size reduction will lead to the reduction of $\mathrm{Mn}-\mathrm{O}$ bond lengths. As a consequence of bond length modifications, the system inhibits the formation of charge ordered phase. These two factors stabilize the insulating phase for all the thicknesses as observed in electrical transport measurements.

It is well known that the increase of Jahn-Teller strain facilitates the formation of polarons by providing strong 
electron-phonon coupling and as a consequence, the polaron binding energy increases. Jahn-Teller strain $\left(\varepsilon_{\mathrm{J}-\mathrm{T}}\right)$ is evaluated by $\sqrt{ } 1 / 6\left(2 \varepsilon_{\mathrm{zz}}-\varepsilon_{\mathrm{xx}}-\varepsilon_{\mathrm{yy}}\right)$, where $\varepsilon_{\mathrm{zz}}$ is out-ofplane and $\varepsilon_{\mathrm{xx}}$ and $\varepsilon_{\mathrm{yy}}$ are in-plane strain components [11] and is shown in Fig. 3.

Despite there is not a distinct trend observed on $\varepsilon_{\mathrm{J}-\mathrm{T}}$, one can observe a decrease of Jahn-Teller strain with increase of film thickness. This feature reflects a reduced contribution of $\varepsilon_{\mathrm{J}-\mathrm{T}}$ especially for the higher order thickness, which demonstrates the weak strain approach as a result of strain relaxation upon increasing film thickness. This characteristic nature correlates well with the decreasing values of polaron binding energy as shown in Table 1.

Table 1. Influence of thickness on polaronic formation energy.

\begin{tabular}{cc}
\hline $\begin{array}{c}\text { Thickness } \\
(\AA)\end{array}$ & $\begin{array}{c}\text { Binding energy } \\
(\mathrm{eV})\end{array}$ \\
\hline 400 & 0.1291 \\
800 & 0.1421 \\
1000 & 0.1405 \\
1600 & 0.1281 \\
2000 & 0.1182 \\
\hline
\end{tabular}

The appearance of charge-ordered phase (CO) for 200 $\mathrm{nm}$ is presumably due to reduced Jahn-Teller strain. However, such a charge ordered phase was not observed for thinner films due to pronounced $\varepsilon_{\mathrm{J}-\mathrm{T}}$.

Temperature dependent electrical transport measurement was performed with the four-probe method, Fig. 4. The measured resistivity data shows distinct differences for various thicknesses and the data clearly evidences an insulating feature. It is generally regarded that the insulator like behavior below $\mathrm{T}_{\mathrm{c}}$ is associated with the existence of polaronic regime [12].

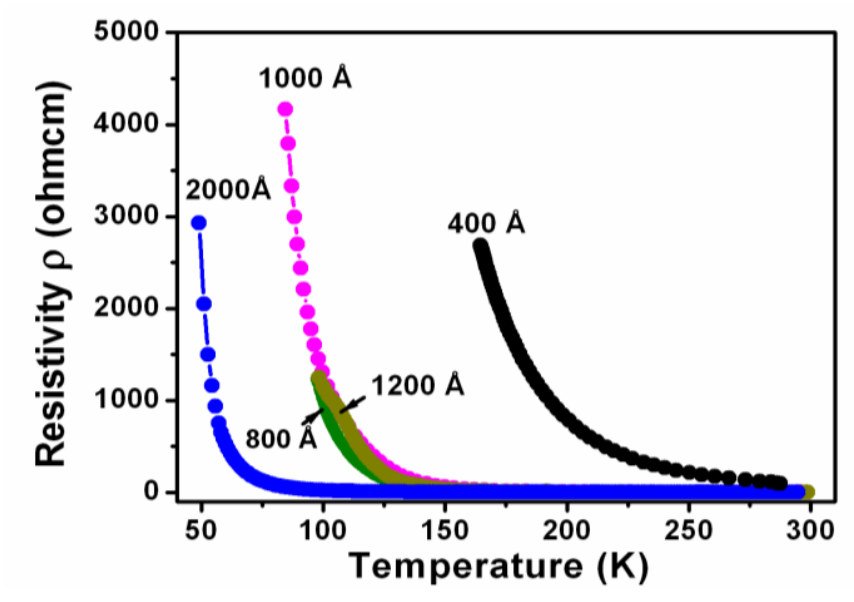

Fig. 4. Resistivity versus temperature graphs for $100 \mathrm{~nm}$ and $200 \mathrm{~nm}$ thicknesses of the $\mathrm{La}_{0.9} \mathrm{Ca}_{0.1} \mathrm{MnO}_{3}$ films.

Further, the manganites act upon the preconditions of polaron formation namely large electron-lattice coupling and low hopping rates [13]. It is well established that in the $\mathrm{PM}$ regime the localized electronic charge carrier concentration remains constant follows linearity in the
Arrhenius plot due to adiabatic hopping motion of small polarons. Such a behavior was observed for the $\ln (\rho / T)$ versus inverse temperature, which is evidenced from Fig. 5.

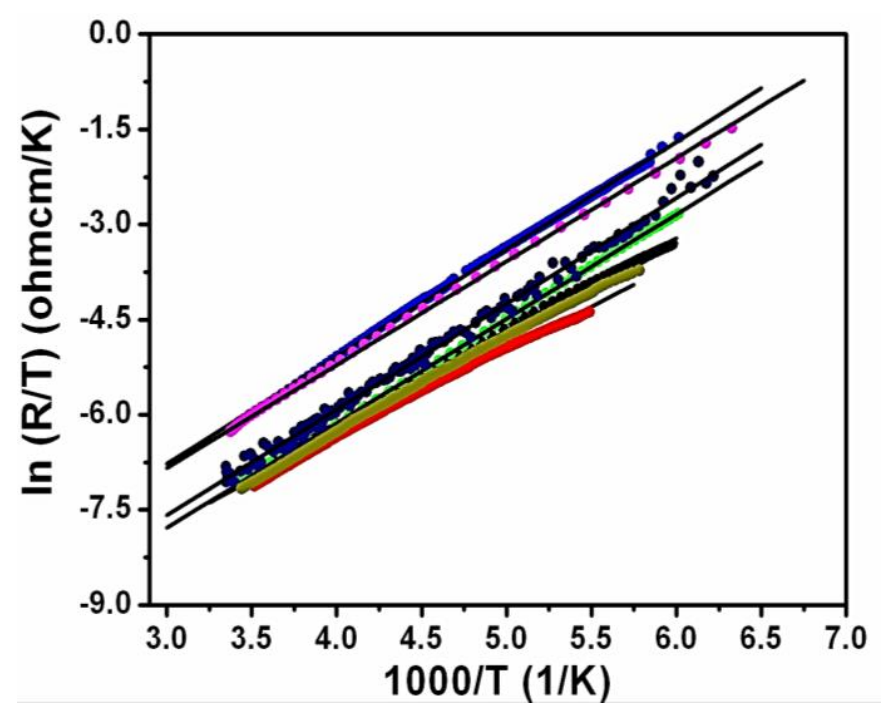

Fig. 5. Plot of $\ln (\rho / T)$ versus inverse temperature $100 \mathrm{~nm}$ and $200 \mathrm{~nm}$ thicknesses of the $\mathrm{La}_{0.9} \mathrm{Ca}_{0.1} \mathrm{MnO}_{3}$ films.

This linear behavior gives evidence for the adiabatic small polaron conduction mechanism which has been already well ascribed in $\mathrm{La}_{1-\mathrm{x}} \mathrm{Ca}_{\mathrm{x}} \mathrm{MnO}_{3}$ films. The electrical resistivity as a result of hopping of adiabatic small polarons is given by;

$$
\rho=A T \exp \left(E_{A} / k_{B} T\right)
$$

where the activation energy, $\mathrm{E}_{\mathrm{A}}=\mathrm{Ep} / 2+\varepsilon_{0}-\mathrm{J}, \varepsilon_{\mathrm{o}}$ is the energy required to generate intrinsic carriers and $J$ is the transfer integral, $\mathrm{k}_{\mathrm{B}}$ is the Boltzmann constant and the prefactor ' $A$ ' depends on the polaronic concentration, hopping distance and the frequency of the longitudinal optical phonon. Values of $\mathrm{A}$ and $\mathrm{E}_{\mathrm{A}}$ are obtained from the linear fit to the above equation. In the adiabatic limit, the role of $\varepsilon_{o}$ and $\mathbf{J}$ may be neglected, therefore the polaronic formation energy is given by $\mathrm{E}_{\mathrm{p}}=2 \mathrm{EA}$.

Table 1 shows the thickness dependence on polaronic formation energy. From the table it is clear that the polaronic formation energy $\left(\mathrm{E}_{\mathrm{p}}\right)$ decreases with increasing thickness. On comparing with our previous results on $\mathrm{La}_{0.9} \mathrm{Sr}_{0.1} \mathrm{MnO}_{3}$ [9], an opposite trend in polaronic formation energy $\left(E_{p}\right)$ with $10 \%$ increase of $E_{p}$ values was observed. The observed difference in $\mathrm{E}_{\mathrm{p}}$ is due to the different strain states exhibited by $\mathrm{La}_{0.9} \mathrm{Ca}_{0.1} \mathrm{MnO}_{3}$ (tensile) and $\mathrm{La}_{0.9} \mathrm{Sr}_{0.1} \mathrm{MnO}_{3}$ (compressive). Hence it should be emphasized that the electron-phonon coupling dominates the strain effect through the modifications of Mn-O-Mn bond angle and $\mathrm{Mn}-\mathrm{O}$ bond lengths.

\section{Conclusion}

The structural and transport properties of $\mathrm{La}_{0 .} \mathrm{Ca}_{0.1} \mathrm{MnO}_{3}$ films grown on $\mathrm{SrTiO}_{3}$ substrate employing pulsed laser deposition technique have been investigated. Structural study by X-ray diffraction technique confirms the single phase nature with (0b0) orientation. The observed 
reduction of orthorhombic distortion and unit cell volume leads to the enhancement of Mn-O-Mn bong angle closer to the ideal value. The reduced trend observed for the Jahnteller strain correlates well with the observed polaronic binding values. In the limit of adiabatic approximation at higher temperature, the transport properties of various thicknesses obey the small polaron hopping conductivity. The polaronic formation energy decreases with increasing film thickness as contrary to $\mathrm{La}_{0.9} \mathrm{Sr}_{0.1} \mathrm{MnO}_{3}$ films due to different strain states.

\section{Reference}

1. Colossal Magnetoresistive Oxides, edited by Y.Tokura, Gordon \& Breach, New York, 2000

2. Zener, C. Phys. Rev. 1951, 82, 403

DOI: $10.1103 /$ PhysRev.82.403

3. Tanasescu, S.; Maxim, F.; Teodorescu, F.; Giurgiu, L. J. Nanosci. Nanotechnol. 2008, 8, 914.

DOI: $10.1166 /$ jnn.2008.D127

4. Zhao, G.; Conder, K.; Keller, H.; Muller, K.A. Nature 1996, 381, 676. DOI: $10.1038 / 381676 \mathrm{a} 0$

5. Billinge, S.J.L.; Difrancesco, R.G.; Kwei, G.H.; Neumeier, J.J.; Thompson, J.D. Phys. Rev. Lett. 1996, 77, 715. DOI: $\underline{10.1103 / \text { PhysRevLett. } 77.715}$

6. Jaime, M.; Salamon, M.B.; Pettit, K.; Rubinstein, M.; Treece, R.E. ; Horwitz, J.S. ; Chrisey, D.B. Appl. Phys. Lett. 1996, 68, 1576. DOI: $\underline{10.1063 / 1.116686}$
7. Song, X.F.; Lian, G.J.; Xiong, G.C. Phys. Rev. B 2005, 71, 214427. DOI: $10.1103 /$ PhysRevB.71.214427

8. Tovstolytkin, A.I.; Gorkov, D.V.; Matvienko, A.I. Low Temp. Phys. 2008, 34, 192.

DOI: $10.1063 / 1.2888758$

9. Chen, X.J.; Soltan, S.; Zhang, H.; Habermeier, H.U. Phys. Rev. B 2002, 65,174402

DOI: $10.1103 /$ PhysRevB.65.174402

10. Cullity, B.D.; Stock, S.R.; Elements of X-ray Diffraction, 3rd ed., Prentice. Hall, New Jersey, 2001, p. 363. 2480.

11. Aydogdu, G.H.; Kuru, Y.; Habermeier, H.U. Mater. Sci. Eng. B 2007, $144,123$. DOI: $10.1016 /$ j.mseb.2007.07.016

12. Ibarra, M.R.; Algarabel, P.A.; Marquin, C.; Blasco, J.; Garcia, J. Phys. Rev. Lett. 1995, 75, 3541 DOI: 10.1103/PhysRevLett.75.3541

13. MIllis, A.J.; Littlewood, P.B.; Shraiman, B.I. Phys. Rev. Lett. 1995 , $74,5144$.

DOI: $10.1103 /$ PhysRevLett.74.5144

\section{Advanced Materials Letters}

\section{Publish your article in this journal}

ADVANCED MATERIALS Letters is an international journal published quarterly. The journal is intended to provide top-quality peer-reviewed research papers in the fascinating field of materials science particularly in the area of structure, synthesis and processing, characterization, advanced-state properties, and applications of materials. All articles are indexed on various databases including $\mathrm{DOAJ}$ and are available for download for free. The manuscript management system is completely electronic and has fast and fair peer-review process. The journal includes review articles, research articles, notes, letter to editor and short communications.
JOURNAL

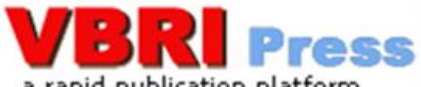

a rapid publication platform

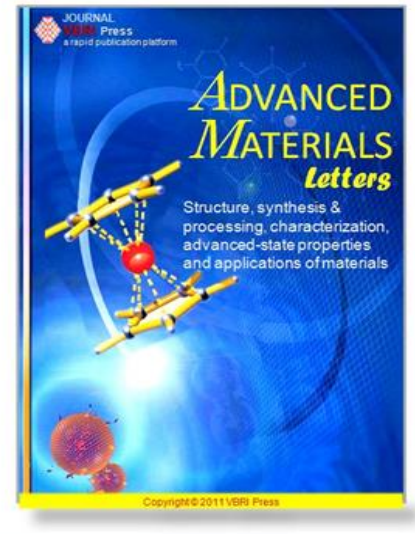

